\title{
Investment Bank Risk Prediction Model Based on Dynamic Parameter Neural Network
}

\author{
Yixin Zhang \\ School of Economics, Nankai University, Tianjin, 300071, China
}

Keywords: Investment banking; Risk prediction; Momentum factor

\begin{abstract}
For traditional neural network algorithm to predict the risk in the investment banking applications exhibit, predictive accuracy is not high. In this paper, a dynamic parameter optimization of investment banking based on neural network, is the first risk prediction model under the dynamic consolidation and deletion rules, and adaptive dynamic adjustment of parameters to obtain the most appropriate neural network model, then in order to accelerate convergence and prevent oscillation, the introduction of a momentum factor, last modified error function, to ensure the network training error as small as possible, so the network has a smaller case weights. The simulation results showed that the bank's risk prediction model based on neural network optimized dynamic parameters proposed, compared to standard neural network algorithm, has higher prediction accuracy.
\end{abstract}

\section{Introduction}

As the trend of global economic integration intensifies, the financial industry's operations in the entire economic system become more apparent. If there is any problem in one link, it will have a certain impact on the entire industry. Finance is the core of the modern economy. The safe operation of the financial system is related to the healthy development of the economy and social stability. The inherent vulnerability of investment banks and the excessive volatility of financial asset prices such as securities have led to the risk of investment banks being a risk complex.

China's investment banks are still in the initial stage of development, and the risk warning research on investment banks is also in a relatively early stage. However, the traditional economic early warning model has the difficulty of dealing with highly nonlinear models, overcoming the indirect, time-consuming, and inefficient limitations of lack of adaptive capabilities, information and knowledge acquisition. For example, a certain crisis in the credit situation of financial institutions will lead to a certain decline in the quality of credit assets, which will lead to a surge in non-performing loans and bad debts of banks, and even lead to bankruptcy, which will bring serious blows to China's national economy. Therefore, under such a social background, the establishment of a bank credit risk early warning system to avoid the occurrence of bank credit risk has gradually become a social hotspot of financial theory, practice and government.

\section{Investment Bank Risk Analysis}

\subsection{Risk classification of investment banks}

Investment bank risks can be divided into different types according to different standards. According to Markowitz and Sharp's modern investment theory, investment bank risks can be divided into two categories: systemic risks and non-systemic risks.

\subsubsection{Legal and regulatory risks}

Legal risk arises from the possibility that one party to the transaction cannot perform the contract with the other party. The legal risk may arise because the contract is not enforced at all, or the party to the contract exceeds the statutory authority. 


\subsubsection{Market risk}

Market risk refers to the possibility that a financial company's financial assets will be lost due to adverse fluctuations in market system factors. These factors include interest rates, exchange rates, stock indices, and commodity prices. System factors are included in all aspects of the market, so market risks affect the profit and loss of each business of the securities company.

\subsubsection{Operational risk}

Operational risk refers to the possibility of losses caused by investment banks in the course of business operations and management, including investment bank decision-making risk, internal control risk and technical risk.

\subsubsection{Credit risk}

Credit risk refers to the possibility of losses due to the credit crisis, including loans, swaps, options and the risk of loss from counterparty defaults during the settlement process. The degree of credit risk and the nature of the transaction have a lot to do with the credit system.

\subsubsection{Liquidity risk}

Liquidity risk, also known as liquidity risk, refers to the fact that investment banks are unreasonable in asset structure, their liquidity ratio is too low, their financial structure lacks liquidity, financial products cannot be liquidated and positions are tight, making investment banks unable to repay debts. risks of.

\subsection{Investment Bank Risk Assessment Theory}

After the traditional subjective analysis and financial ratio analysis artificial intelligence application process, the bank's risk assessment theory is developing continuously. At present, a relatively modern risk quantitative management model has emerged. The signal method used by Kaminsky et al. is used to predict the turning point of the business cycle in advance, and after being scientifically researched by experts such as Ka-minsky, it is mainly used in the evaluation and early warning of financial crisis management. Li Meng and Chen Liuqin mainly studied the listed company as a standard for judging credit risk after it failed to repay the bank loan amount on time. Combining the principal component analysis method and the artificial neural network scheme, the model of credit risk measurement of commercial banks in China is established. Under the in-depth study and research, the financial information and default data of commercial bank loan customers in a certain country are used as analysis samples. Dong Aiyang proposed that the commercial bank has entered the period of comprehensive management of assets and liabilities. In order to prevent the risk of liquidity, some bank management indicators were selected to construct the BP artificial neural network model, and the sample data $\mathrm{O} 2=\omega=\mu(\mathrm{x})$ Perform network training operations. Chen Qingguang et al. used the neural network model to measure and measure the operational risk of commercial banks on the basis of domestic scholars' operational risk measurement methods. When Zhou Enhong and others proposed to study the operational risks of commercial banks, it is more scientific to select the sample data type when selecting the model tool of the neural network method. Chen Chaozhen used the BP neural network scheme to establish a risk pre-test model for commercial banks in China, and used the risk warning indicators of commercial banks as input values. The output value indicates the early warning degree of commercial banks in China.

\section{Neural network based predictive model}

For the pre-test of risk of investment banks, the most used one at this stage is the neural network model. The construction method of the model is as follows:

Tier 0: The parameters in the transmission of the investment bank risk prediction model are taken as the values of each neuron in the fuzzy neural network, that is, the input values of the neurons.

$$
O_{i}^{o}=x_{i}
$$


The number of neurons is the number of references to be passed by the pre-test model in investment bank risk.

Tier 1: That is, to calculate the membership of each input parameter corresponding to each fuzzy set. The output $\mathrm{O} 1$ of the $\mathrm{i}$-th neuron in layer 1 is calculated by:

$$
O_{i}^{i}=\mu_{A_{k}^{i k}}\left(x_{k}\right)
$$

Where xk is the input value. The total number of neurons is the total number of fuzzy sets. In general, the value of the membership function $\mu \mathrm{Aik}$ (xk) is expressed as a bell membership function or a Gaussian membership function:

$$
\mu_{A_{k}^{i k}}\left(x_{k}\right)=\frac{1}{1+\left[\left(-\frac{x-c_{i}}{a_{i}}\right)^{2}\right]}
$$

Or

$$
\mu_{A_{k}^{i k}}\left(x_{k}\right)=\exp \left\{-\left(\frac{x-c_{i}}{a_{i}}\right)^{2}\right\}
$$

Layer 2: Each neuron is a fixed neuron labeled $\prod$, whose output is the product of all parameter input signals:

$$
O_{i}^{2}=\omega_{i}=\prod_{k=1}^{n} \mu_{A_{k}^{i k}}\left(x_{k}\right)
$$

In the above formula, the input of the i-th parameter neuron is equal to the pre-requisite of the $\mathrm{i}$-th risk pre-detection, and the output value is the indicator of the risk pre-detection. Where $\mathrm{i}=1$, $2, \ldots, \mathrm{m}, \mathrm{m}$ are the number of risk prediction indicators.

Layer 3: Calculating the output

$$
O_{i}^{3}=\omega_{i} f_{i}=\omega_{i}\left(p_{1}^{i} x_{1}+p_{2}^{i} x_{2}+\ldots+p_{n}^{i} x_{n}+q^{i}\right)
$$

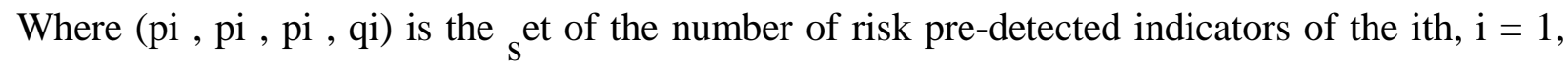
$2, \ldots, \mathrm{m}$.

Layer 4: Summation

$$
O_{i}^{4}=\sum_{k=1}^{m} O_{k}^{3}, i=1
$$

Layer5: Normalization

$$
O_{i}^{5}=\frac{O_{i}^{4}}{\sum_{k=1}^{m} \omega_{k}}, i=1
$$

According to the above discussion, even if the investment bank model can be successfully detected in advance, the short board of the neural network itself has not been fully processed. Therefore, it is relatively simple to optimize the operation of the neural network when the data is large and enter the local optimal problem.

\section{Dynamic Parameter Optimization of Neural Networks}

A neural network is a network of massively parallel units that are composed of a large number of simple units and hierarchical organizations of these units. The neural network solves the problem by "training": Using the same series of input factors and ideal output factors as "samples", the network 
is adequately trained according to certain algorithms, so that the neural network can learn the basics contained in the "solution" principle. When the training is complete, the model can be used to solve similar problems.

\subsection{Parameter adaptive adjustment}

For the pre-test of risk of investment banks, the most used one at this stage is the neural network model. The construction method of the model is as follows:

Layer 0: The parameters in the transmission of the investment bank risk prediction model are taken as the values of each neuron in the fuzzy neural network, that is, the input values of the neurons.

As long as there are relatively many nodes in the invisible neural network, they can be close to the nonlinear function. Specifically, the choice of hidden nodes is more difficult. If there are fewer hidden nodes, there is no convergence. If there are more hidden nodes, the network performance will be worse. This requires us to find the best number of hidden nodes. The most suitable solution is to use the network to make corresponding adjustments, and finally get the optimal neural network model. Suppose Opi is expressed as the state of the hidden node $\mathrm{i}$ at the pth sample, Opj is expressed as the output of the hidden $\mathrm{j}$ at the pth sample, and $\mathrm{N}$ is the total number of samples, then:

$$
\bar{O}_{i}=\frac{1}{N} \sum_{p=1}^{N} O_{p i}, \bar{O}_{j}=\frac{1}{N} \sum_{p=1}^{N} O_{p j}
$$

Let

$$
\begin{array}{r}
x_{p}=O_{p i}-\frac{1}{N} \sum_{p=1}^{N} O_{p i}=O_{p i}-\bar{O} \\
y_{p}=O_{p j}-\frac{1}{N} \sum_{p=1}^{N} O_{p j}=O_{P J}-\overline{O_{J}}
\end{array}
$$

Then the relationship between the sequence Opi and Ipj is:

$$
R_{i j}=\frac{\sum_{p=1}^{n} x_{p} y_{p}}{\sqrt{\sum_{p=1}^{N} x_{p}^{2} \sqrt{\sum_{p=1}^{N} y_{p}^{2}}}}
$$

Then you can get $|\mathrm{Rij}| \leq 1$.

The closer Rij is to 1 means that the Opi and Opj sequences are closely related and the relative dispersion is smaller. Conversely, the correlation between the two is larger and the dispersion is also larger.

Therefore, the following two inferences can be obtained:

The correlation coefficient of the hidden nodes $\mathrm{i}$ and $\mathrm{j}$ in the same layer is set to Rij , and Rij represents the correlation between the two nodes $\mathrm{i}$ and $\mathrm{j}$. If Rij is large, it means that the sexual nodes of $i$ and $j$ are duplicated, and compression is required.

The divergence of the sample $\mathrm{Si}, \mathrm{Si}=\sum \mathrm{Opi}-\mathrm{Oi}$, if $\mathrm{S}$ is too small, then the hidden node i does not have a large change in the output value, and will not affect the network, so it can be deleted.

According to the above inference, the dynamic merge and cut-off rules proposed in this paper:

(1) If $|\mathrm{Rij}| \geq \mathrm{C} 1$ and meets $\mathrm{Sj} \geq \mathrm{C} 2$, the same-level hidden nodes $\mathrm{i}$ and $\mathrm{j}$ can be merged. Where $\mathrm{C} 1$ and $\mathrm{C} 2$ are the specified lower limits, generally the value range of $\mathrm{C} 1$ is $0.8 \sim 0.9$, and $\mathrm{C} 2$ is $0.001 \sim 0.01$.

(2) If $\mathrm{Si}<\mathrm{C} 2$, then node i acts as a domain value and can be merged with the threshold, then node i can be deleted.

\subsection{Error correction}

The neural network algorithm performs deductive reasoning under the premise of a certain 
gradient method. In the ordinary optimal gradient method, the step sizenis obtained according to the one-dimensional search, and the solution is as follows:

(1) The point $\mathrm{w}(\mathrm{o})$ of the initial weight and the allowable error $\varepsilon<0$

(2) Negative gradient direction of the calculation error $E(n)=-\Delta E(w)$

(3) If $\mathrm{d}(\mathrm{n})<\varepsilon$, then the calculation is stopped; otherwise, starting from $\mathrm{w}(\mathrm{n} 0)$, one-dimensional search is performed along $d(n)$ to find the optimal step size $\eta(n 0)$.

$$
E_{\text {total }}\left[\omega\left(n_{o}\right)+\eta\left(n_{o}\right) d\left(n_{o}\right)\right]=\min E_{\text {total }}\left[\omega\left(n_{o}\right)+d\left(n_{o}\right)\right]
$$

(4) Iteration of the right to exercise:

$$
w\left(n_{0}+1\right)=w\left(n_{0}\right)+\eta\left(n_{0}\right) d\left(n_{0}\right)
$$

Then go to (2) and make some adjustm $\mathrm{e}^{\text {nts }}$ to the step size,

$$
\begin{gathered}
\eta=\mu \varphi, \varphi>1, \text { when } \Delta E<0 \\
\eta=\mu \beta, \beta>1, \text { when } \Delta E<0
\end{gathered}
$$

Here, $\varphi$ and $\beta$ represent constants, and $\Delta \mathrm{E}=\mathrm{E}$ total $(\mathrm{n} 0)$ - $\mathrm{E}$ total (n0 - 1). Of course, the variable step size can also be performed by other methods.

After getting the step size accurately, you get an iterative formula:

$$
\omega\left(n_{0}+1\right)=\omega\left(n_{0}\right)+\eta\left(n_{0}\right) d\left(n_{0}\right)
$$

In order to accelerate convergence and prevent the occurrence of oscillation, a momentum factor $\alpha$ is introduced to represent the momentum term, which is a positive number.

The third term means the modification of the right at the moment of memory, and the direction of modification at time (n0) is (n0 - 1).

The combination of the direction of the moment and the direction of the $\left(\mathrm{n}_{0}\right)$ moment. Formula (16)

$$
\begin{aligned}
\omega\left(n_{0}+1\right)= & \omega\left(n_{0}\right)+\eta\left(n_{0}\right) d\left(n_{0}\right)+\alpha \Delta w\left(n_{0}\right) \\
& \omega\left(n_{0}+1\right)=\omega\left(n_{0}\right)+\eta\left(n_{0}\right)\left[d\left(n_{0}\right)+\frac{\alpha}{\eta\left(n_{0}\right)}\right] \Delta w\left(n_{0}\right) \\
= & \omega\left(n_{0}\right)+\eta\left(n_{0}\right)\left[d\left(n_{0}\right)+\frac{\alpha \eta\left(n_{0}-1\right)}{\eta\left(n_{0}\right)} d\left(n_{0}-1\right)\right]_{(17)}
\end{aligned}
$$

The form of the above formula is similar to the equation of the conjugate gradient method, but here $d(n 0-1)$ and $d(n 0)$ do not have a conjugate relationship, but $0<\alpha<1$. So it is recommended to perform at $\eta(\mathrm{n} 0)$ when adjusting, you will encounter $\Delta \mathrm{E}>0$. When $\eta$ is to be reduced, let $\alpha=0$, then adjust to $\eta$ to increase $\alpha$ to 0 .

In summary, the new function indicator function can ensure that the network training error is reduced and the network has a smaller value. The effect is equivalent to automatically reducing the network specifications.

\section{Simulation and verification}

In order to test the effective ability of the improved algorithm proposed in this paper, this paper takes the actual data of an investment bank as a practical example, and uses the standard neural network algorithm and the improved neural network algorithm mentioned in this paper to pre-detect the risk. The simulation experiment results are as follows: 

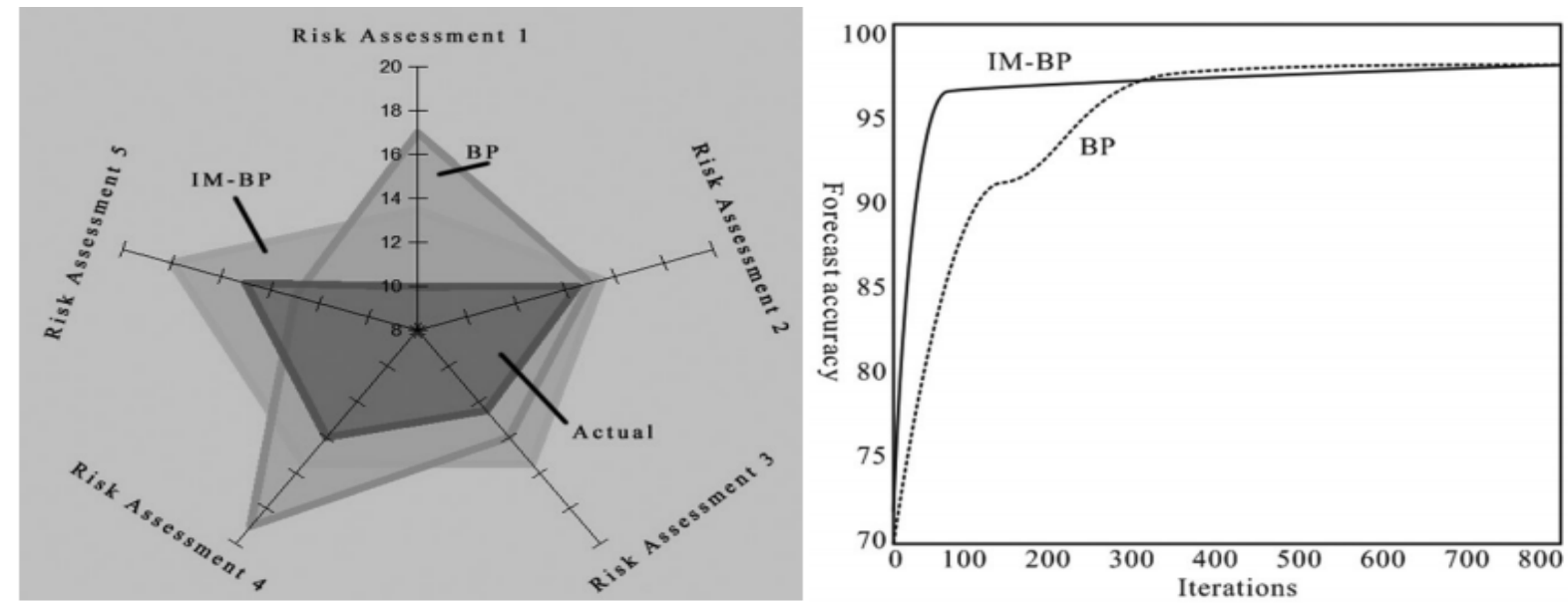

Investment bank risk prediction

Both algorithms compare the predictive accuracy

According to the experimental results of the simulation, it can be seen that the improved algorithm has relatively good pre-detection accuracy due to the dynamic adaptive adjustment of parameters and certain error correction operations for the standard algorithm.

\section{Conclusion}

At the current stage of economic globalization, domestic market interest rates are also rising, the volatility of financial markets is gradually increasing, and commercial banks are facing a credit crisis. Therefore, based on the actual situation of investment banks, this paper proposes a pre-detection model of investment bank risk based on dynamic parameter optimization neural network. According to the simulation experiment results, the improved algorithm proposed in this paper has higher pre-detection accuracy.

\section{References}

[1] Wang X. Model of Investment Risk Prediction Based on Neural Network and Data Mining Technique for Construction Project[M]. IEEE Computer Society, 2008.

[2] Zhang X, Xiao L, Kang J. Degradation prediction model based on a neural network with dynamic windows[J]. Sensors, 2015, 15(3):6996.

[3] Guan X, Guo M, Cao J. Study on wood dyeing pigment formula prediction model based on dynamic fuzzy neural network[C]// International Conference on Computer Engineering and Technology. IEEE, 2010:V3-423-V3-427.

[4] Wen K. Investment Bank Risk Prediction Model of Dynamic Parameters of the Neural Network[J]. Bulletin of Science \& Technology, 2015.

[5] Guan X, Guo M, Cao J. Study on wood dyeing pigment formula prediction model based on dynamic fuzzy neural network[C]// International Conference on Computer Engineering and Technology. IEEE, 2010:V3-423-V3-427. 